Referencia para citar este artículo: Salva-Mut, F., Nadal-Cavaller, J. \& Melià-Barceló, M. A. (2016). Itinerarios de éxito y rupturas en la educación de segunda oportunidad. Revista Latinoamericana de Ciencias Sociales, Niñez y Juventud, 14 (2), pp. 1405-1419.

\title{
Itinerarios de éxito y rupturas en la educación de segunda oportunidad*
}

\author{
FRANCESCA $S_{A L V A-M U T}{ }^{* *}$ \\ Profesora Universidad Islas Baleares, España. \\ JOAN NADAL-CAVALLER ${ }^{* * *}$ \\ Investigador Universidad de las Islas Baleares, España. \\ MARIA AGNÈS MELIÀ-BARCELO $\hat{O}^{\text {****}}$ \\ Profesora Universidad de las Islas Baleares, España.
}

\section{Artículo recibido en noviembre 25 de 2015; artículo aceptado en marzo 13 de 2016 (Eds.)}

- Resumen (descriptivo): El concepto "educación de segunda oportunidad" (en adelante Esop) hace referencia a los dispositivos puestos en marcha para luchar contra la exclusión social de aquellas personas (en particular los jóvenes) que han abandonado los estudios con un nivel educativo inferior al de secundaria postobligatoria. Los programas de formación para el empleo dirigidos a jóvenes son los dispositivos de Esop más extendidos en España.

En este artículo, se presentan los resultados de una investigación evaluativa de uno de estos programas basada en el método biográfico. El abordaje integral y la individualización, los contenidos de la formación y los aprendizajes realizados, las estrategias metodológicas, el vínculo afectivo y el entorno educativo se manifiestan como determinantes del éxito. También queda patente el impacto positivo de la participación a nivel personal y laboral.

Palabras clave: Jóvenes sin cualificación, transición de la escuela al trabajo (Tesauro Europeo de los Sistemas Educativos); formación profesional, deserción escolar, factores de éxito (Tesauro de la Educación de Unesco-OIE).

\section{Successful and unsuccessful pathways in second chance education programmes}

- Abstract (descriptive): The concept of second chance education (hereafter SCE) refers to the programs launched to combat the social exclusion of people (in particular young people) who have left school with a level of education below upper secondary. Training employment programs aimed at young people are the most widespread SCEs in Spain.

The results of an evaluative investigation of one of those programs, based on the biographical method, are shared in this article. A comprehensive educational approach that includes its

\footnotetext{
Este artículo de investigación científica y tecnológica se basa en la investigación "Condiciones de éxito y rupturas en la participación de la población joven en los dispositivos de educación de segunda oportunidad: el caso del CFO Jovent", que se ha desarrollado entre enero, 2009 y diciembre, 2013. La investigación ha recibido financiación del Ayuntamiento de Palma de Mallorca, en fecha de 15 de enero de 2009 (ref. 20093036). El artículo pertenece al área de conocimiento Ciencias de la Educación y a la subárea Educación Especial.

** Doctora en Ciencias de la Educación. Profesora Titular del Departamento de Pedagogía Aplicada y Psicología de la Educación de la Universidad de las Islas Baleares. Correo electrónico: f.salva@uib.es

*** Licenciado en Ciencias de la Educación. Colaborador de investigación. Personal Técnico de la Universidad de las Islas Baleares. Correo electrónico: ocds.tecnic@uib.es

**** Licenciada en Ciencias de la Educación. Profesora Asociada del Departamento de Pedagogía Aplicada y Psicología de la Educación. de la Universidad de las Islas Baleares. Correo electrónico: ma.melia@uib.es
} 
individualization, training content and the learnings achieved, methodological strategies, emotional ties and the educational environment are identified as determinants of success. Moreover, there is a noticeable positive impact of the young people's participation on their personal lives and work opportunities.

Key words: Unqualified young people, transition from school to work (Thesaurus for Education Systems in Europe); vocational training, dropping out, success factors (Unesco-IBE Education Thesaurus-OIE).

\section{Itinerários de sucesso e de rupturas na educação de segunda oportunidade}

- Resumo (descritivo): O conceito de "educação de segunda oportunidade" (Esop) faz referência aos dispositivos colocados em funcionamento para lutar contra a exclusão social das pessoas (em particular os jovens) que tenham abandonado os estudos com um nível educativo inferior ao do ensino secundário superior. Os programas de formação profissional dirigidos aos jovens são os dispositivos de Esop mais presentes na Espanha.

Neste artigo, apresentam-se os resultados de uma pesquisa avaliativa de um desses programas, com base no método biográfico. A abordagem integrada e a individualização, o conteúdo da formação e os aprendizados obtidos, as estratégias metodológicas, o vínculo afetivo e o ambiente educacional se manifestam como fatores determinantes do sucesso. O impacto positivo da participação a nível pessoal e profissional também é evidente.

Palavras-chave: Jovens sem qualificação, transição da escola para o trabalho (Thesaurus Europeu dos Sistemas Educativos); formação profissional, abandono escolar, fatores de sucesso (Thesaurus de Educação da Unesco-OIE).

\section{-1. Introducción. -2. Metodología. -3. Resultados. -4. Conclusiones. -Lista de referencias.}

\section{Introducción}

En España, uno de cada cuatro jóvenes abandona la Educación Secundaria Obligatoria (ESO) sin obtener la titulación de Graduado/a en Educación Secundaria Obligatoria (Geso) y de estos son muy pocos los que posteriormente consiguen obtenerla (Albert, Davia \& Toharia, 2008, García, Casal, Merino \& Sánchez, 2013). Por otra parte, los jóvenes sin titulación encuentran dificultades para participar en la formación (Albert et al., 2008, FernándezEnguita, Mena \& Riviere, 2010, García et al., 2013, Salvà-Mut, Quintana-Murci \& Desmarais, 2015) y en el mercado laboral (García-Montalvo \& Peiró, 2011, OECD, 2013); dificultades que ponen de relieve la existencia de una estrecha relación entre la exclusión educativa y la exclusión social (Sen, 2000, Silver \& Miller, 2003, Albert \& Davia, 2011).

En Europa, el concepto de Educación de SegundaOportunidad(Esop) empezó a utilizarse en el marco de las estrategias encaminadas a combatir la exclusión puestas en marcha de acuerdo con las orientaciones del Libro Blanco sobre la educación y la formación "Enseñar y aprender-hacia la sociedad cognitiva" (Comisión de las Comunidades Europeas, 1995). En este contexto, la Esop, desarrollada inicialmente a través del programa Second Chance Schools, promueve nuevas formas de educación y formación dirigidas a jóvenes con un nivel educativo que les impide continuar su formación o encontrar un empleo (European Commission, 2001). Su objetivo es facilitar la inserción social y profesional de dichos jóvenes a través de una amplia gama de oportunidades formativas adaptadas a sus necesidades, entre las que figuran los programas de formación para el empleo.

Cabe constatar que la investigación sobre Esop es escasa y a pesar de los avances (European Union, 2013), el conocimiento sobre las condiciones y los factores de éxito de estos dispositivos es todavía insuficiente (SalvàMut \& Sureda-Negre, 2012); se trata pues de un ámbito falto de evidencias que requiere de la generación de conocimiento que propicie la mejora y la innovación de las prácticas educativas. Los trabajos realizados en España remarcan un amplio consenso sobre la necesidad de mejorar la calidad de la oferta formativa 
existente, ajustándola a las características y necesidades de los colectivos destinatarios y de los territorios; también destacan el elevado grado de compromiso de los profesionales $\mathrm{y}$ entidades implicadas (Salvà-Mut, OliverTrobat \& Calvo-Sastre, 2006).

Marhuenda y Navas (2004) señalan, como sus aspectos positivos la flexibilidad y la pluralidad de agentes promotores, y alertan sobre la precariedad de estos programas debido a una falta de financiación estable. Centrándose en los aspectos pedagógicos, Marhuenda, Navas y Pinazo (2004) destacan la creación de un clima de aula más favorable al aprendizaje y una "aproximación educativa" más que "sancionadora o punitiva" con un componente de empatía emocional del profesorado en relación con el alumnado. Entre los elementos facilitadores destacan el papel de la tutoría, la flexibilidad organizativa, el trabajo en equipo de los formadores, la adaptación del currículum y una ratio reducida. En opinión de Salvà-Mut (2009a), para que estos programas sean una oportunidad de éxito para los jóvenes sin titulación, deben tener las siguientes características: alejarse de los modelos escolares tradicionales; plantear situaciones de aprendizaje que promuevan el deseo de aprender; propiciar la construcción de un proyecto personal y profesional propio; evitar la selección inicial del alumnado; prevenir el abandono; ser flexibles respecto a tiempos e itinerarios; conducir a titulaciones reconocidas y relacionadas con certificación académica; facilitar la formación continua; contar con estructuras específicas para facilitar la participación y la perseverancia (servicios de orientación, apoyo y acompañamiento); actuar desde perspectivas globales e integradas (a través de personas y organizaciones del entorno de los jóvenes); vínculo significativo y afectivo entre los jóvenes y los adultos.

En este artículo se presentan los resultados de un trabajo cuya finalidad es conocer las condiciones que llevan a la perseverancia y al éxito o al abandono en los dispositivos ESOP, así como el impacto de la participación en los mismos, con el fin de contribuir a la mejora de estos programas. Para ello, se intenta responder cinco preguntas de investigación: 1) ¿Por qué jóvenes que han abandonado el sistema educativo participan voluntariamente un dispositivo de ESOP? 2) ¿Cuáles son las condiciones que llevan a la perseverancia y al éxito? 3) ¿Cuáles son las condiciones que llevan al abandono antes de finalizar el proceso? 4) ¿Cuáles son las características de los jóvenes que finalizan con éxito? 5) ¿Cuál es el impacto de la participación en el programa?

\section{Metodología}

Se trata de una investigación evaluativa como investigación-acción, con un enfoque biográfico. La investigación evaluativa es pertinente para medir el efecto de los programas y las políticas (Depover, Karsenti \& Komis, 2011) y la perspectiva de la investigación-acción se plantea en el contexto de lo que algunos autores llaman evaluación emancipatoria, que resalta la perspectiva de los participantes en los programas evaluados y que integra la posibilidad de actuar sobre las situaciones estudiadas (Fetterman \& Wandersman, 2005, Miller \& Lennie, 2005, citados en Depover et al., 2011, p. 225).

El enfoque biográfico se plantea desde la perspectiva etnosociológica, cuyo objetivo es "estudiar un fragmento particular de la realidad socio-histórica, un objeto social; comprender como funciona $\mathrm{y}$ como se transforma, poniendo el acento sobre las configuraciones de las relaciones sociales, los mecanismos, los procesos, las lógicas de acción que lo caracterizan" (Bertaux, 1997, p. 7). El estudio de trayectorias sociales es un objeto de estudio clásico de esta perspectiva (Bertaux, 1997, Desmarais, 2009, Argüello, 2014), uno de cuyos referentes es el trabajo sobre transición entre escuela y empleo de Nicole-Drancourt (1991).

La muestra del estudio está formada por un grupo de participantes en el dispositivo de ESOP del Centro de Formación Ocupacional Jovent $^{1}$ titulado "Itinerario integral de orientación, formación e inserción laboral",

Es un centro de iniciativa social, fundado en 1984 con el objetivo de dar respuesta a las elevadas tasas de desempleo juvenil y que se ha convertido en centro de referencia en relación a la formación y la inserción social y profesional de jóvenes sin titulación. Está ubicado en Palma de Mallorca (Baleares, España). Palma de Mallorca es la capital de la comunidad autónoma de las Islas Baleares, una de las diecisiete que conforman el territorio español. Está ubicada en la isla de Mallorca, la mayor del archipiélago, situado al este de la Península Ibérica, en el Mar Mediterráneo. 
dirigido a jóvenes que han salido del sistema educativo formal sin titulación y que presentan factores de vulnerabilidad.

El universo de análisis está formado por 82 jóvenes que en un año causaron baja en el centro; ya sea por la finalización del itinerario o por el abandono del mismo. La selección de la muestra se realizó con los siguientes criterios: Un mínimo de dos personas para cada taller de formación profesional; representación de jóvenes que hubieran finalizado el itinerario y de quienes no lo hubieran finalizado, y de diversas procedencias geográficas. El criterio de género vino determinado por las circunstancias de los talleres: mientras que las personas que habían asistido del taller de estilismo eran mujeres en el resto eran hombres.
La muestra final de informantes fueron quince jóvenes, trece hombres y dos mujeres, de edades comprendidas entre 17 y 24 años, cuya media de edad es 20 años. Su nivel de estudios era inferior al de Graduado/a en Educación Secundaria Obligatoria (GESO), excepto en un caso en que había obtenido dicha titulación. Diez finalizaron el itinerario y cinco lo abandonaron. Respecto a la procedencia geográfica, diez eran españoles y cinco eran inmigrantes (tres habían nacido en África y dos en América del Sur). En la tabla 1 se señalan las principales características de la muestra.

Tabla 1. Características de la muestra.

\begin{tabular}{|c|c|c|c|c|c|}
\hline Nombre $^{* * * * *}$ & E & T & PF/PNF & LN & TM \\
\hline Marta & 20 & Estilismo & PF & España & 19 \\
\hline Rosa & 20 & Estilismo & PF & España & 17 \\
\hline Abdelah & 24 & Carrocería & PF & Argelia & 26 \\
\hline José & 21 & Carrocería & PF & España & 25 \\
\hline Marcial & 20 & Mecánica & PF & España & 25 \\
\hline Mohamed & 22 & Mecánica & PF & Marruecos & 27 \\
\hline Javier & 21 & Mare & PF & Colombia & 30 \\
\hline Tomás & 21 & Mare & PF & España & 27 \\
\hline Gabriel & 19 & Herrería-forja & PF & España & 28 \\
\hline Pedro & 18 & Herrería-forja & PF & España & 24 \\
\hline Juan & 22 & Carrocería & PNF & España & 28 \\
\hline Mustafá & 20 & Herrería-forja & PNF & Mali & 20 \\
\hline Andrés & 20 & Mare & PNF & Colombia & 14 \\
\hline Isidre & 19 & Servtecn & PNF & España & 16 \\
\hline Marc & 17 & Servtecn & PNF & España & 17 \\
\hline
\end{tabular}

${ }^{* * * * *}$ Todos los nombres son ficticios.

E: Edad en el momento de realizar la entrevista. T: Taller. PF: Proceso finalizado. PNF: Proceso no finalizado. LN: Lugar de nacimiento. TM: Tiempo aproximado entre la fecha de salida del programa y la de la entrevista (en meses). Mare: Mantenimiento y reparación de embarcaciones. Servtecn: Servicios técnicos.

El instrumento de recogida de información fue la entrevista biográfica de todos los participantes. El guión de esta se fundamentó en los trabajos de Abdala (2004), Bertrand (2005), Bouder, Cadet y Demazière (1994), Demazière y Dubar (1994), Häfeli y Schellenberg (2009), Salvà-Mut (2007), Salvà-Mut, Calvo y Cloquell (2001) e incluye varios momentos y aspectos de la vida del joven. 
Tabla 2. Dimensiones y variables de la entrevista biográfica

\begin{tabular}{|c|c|}
\hline Dimensiones & Variables \\
\hline Razones de la participación & $\begin{array}{l}\text { - Fuente de información } \\
\text { - Motivos que le llevan a inscribirse }\end{array}$ \\
\hline Biografía escolar & $\begin{array}{l}\text { - Rendimiento académico } \\
\text { - Vinculación emocional con la escuela } \\
\text { - Motivos abandono escolar }\end{array}$ \\
\hline Experiencia dispositivo ESOP & $\begin{array}{l}\text { - Diferencias con la escuela } \\
\text { - Relación con intereses profesionales } \\
\text { - Aprendizajes realizados } \\
\text { - Influencia en diversos aspectos de la vida } \\
\text { - Vinculación afectiva }\end{array}$ \\
\hline $\begin{array}{l}\text { Situación en el momento de la entrevista } \\
\text { y entre este momento y la salida del } \\
\text { dispositivo ESOP }\end{array}$ & $\begin{array}{l}\text { - Situación de convivencia } \\
\text { - Relación con la familia } \\
\text { - Relaciones de pareja } \\
\text { - Amistades } \\
\text { - Actividades que realiza } \\
\text { - Situación laboral } \\
\text { - Valoración de la influencia de su participación en el } \\
\text { dispositivo ESOP en su situación actual }\end{array}$ \\
\hline Perspectivas de futuro & $\begin{array}{l}\text { - Emancipación } \\
\text { - Proyecciones a corto y medio plazo }\end{array}$ \\
\hline
\end{tabular}

Las entrevistas fueron realizadas por dos investigadores, ambos con amplia experiencia, en un espacio cedido por el Ayuntamiento de Palma. Una vez realizadas, se transcribieron y se cumplimentó una plantilla de observaciones que resumía las principales características del lugar donde se había realizado, y los aspectos más significativos del comportamiento de la persona entrevistada. Para realizar el análisis de contenido de las entrevistas, se han seguido las orientaciones propias del análisis de contenido de las entrevistas biográficas, que se describen en la síntesis del proceso (Bertaux, 1997, Desmarais, 2009).

La información recogida en las entrevistas se ha completado con datos del Centro de Formación Ocupacional Jovent (CFO Jovent), tomadas de los archivos, de la base de datos del centro y de entrevistas a sus profesionales. Esta información complementaria se recogió después de realizar las entrevistas y una vez realizada su deconstrucción.

En síntesis, el proceso realizado en relación con los quince casos estudiados fue el siguiente: (1) Entrevista: grabada y transcrita. (2) Deconstrucción mediante la codificación de los contenidos de la entrevista en temas y categorías. (3) Reconstrucción de la entrevista a partir de los temas y categorías. (4) Síntesis de la entrevista a partir de la reconstrucción. (5) Recogida de información del centro y entrevista en profundidad con profesionales de referencia para cada caso. (6) Análisis: a partir de las informaciones recogidas, se da una primera respuesta a las preguntas planteadas en la investigación. Así, para los quince jóvenes se elaboraron cuatro documentos: uno con la entrevista transcrita, otro con la entrevista deconstruida, otro con la síntesis de la entrevista $\mathrm{y}$, finalmente, uno dedicado al análisis.

El proceso de recogida de información, deconstrucción, reconstrucción, síntesis y análisis de la información de cada sujeto fue realizado por una misma persona a partir de los criterios y procedimientos establecidos en las reuniones de equipo. El análisis para cada sujeto fue consensuado por todo el grupo (a partir de la triangulación en el análisis de contenido). Con el objetivo de profundizar en las características de los jóvenes que finalizaron con éxito el itinerario y elaborar una tipología, se llevó a cabo un grupo de discusión formado por los tres investigadores y por un equipo de ocho profesionales del centro, entre los que 
figuraba el profesional de referencia de cada joven $^{2}$.

\section{Resultados}

\section{Las razones para realizar el itinerario}

Hay tres razones principales por las que los jóvenes que abandonaron el sistema educativo sin obtener el título de GESO decidieron participar en el itinerario formativo de Jovent:

(a) La experiencia positiva de personas de su entorno próximo. El conocimiento de esta experiencia les permite considerar que continuar formándose puede facilitar encontrar un empleo. Es la razón más habitual; fue esgrimida por ocho de los quince jóvenes participantes.

(b) La imposibilidad de realizar otra actividad deseada, ya sea formativa o laboral. Cuatro de los quince jóvenes consideran como causa principal de su decisión el no haber conseguido plaza en otro proyecto formativo, no cumplir los requisitos para entrar en un centro de educación de adultos o no haber encontrado empleo.

(c) Una obligación derivada de la situación personal. Las medidas judiciales y el protocolo de atención a menores inmigrantes no acompañados $^{3}$ figuran entre las razones de la participación. Es el caso de dos jóvenes.

El joven que cuando inició el itinerario tenía el título de GESO, es un inmigrante que acudió al centro después de abandonar un Ciclo Formativo de Grado Medio (CFGM).

\section{Las condiciones facilitadoras de la perseverancia y el éxito}

Los jóvenes que finalizaron con éxito enumeran cinco características del programa seguido como sus determinantes: (a) abordaje integral e individualización; (b) contenidos de la formación y aprendizajes; (c) estrategias

2 Profesional que es la persona adulta de referencia del /de la joven en el centro. Ejerce funciones de seguimiento, orientación $\mathrm{y}$ acompañamiento durante todo el proceso.

3 Cuando estos menores cumplen 18 años (mayoría de edad en España) se prioriza su autonomía económica y, por tanto, su participación en el empleo, incluso si están realizando procesos de formación. metodológicas potenciadoras del aprendizaje autónomo y las dinámicas de éxito, con un componente práctico; (d) vínculo afectivo; y (e) entorno educativo. La mayoría (7/10) se refiere a los cinco, mientras los demás (3/10) señalan tres o cuatro.

(a) Sobre el abordaje integral y la individualización. La consideración del joven en su globalidad, que incluye su familia y su entorno, conduce a una individualización del itinerario que se traduce en una adecuación de la duración y de las actividades a la situación de cada participante. Esta adaptación a las necesidades y ritmos de aprendizaje individuales hacen que Rosa, Marta, José y Marcial siguieran procesos largos, de más de 1000 horas, mientras que otros realizaron procesos medios (Mohamed, 660h.) o cortos (Pedro, 313,5 h. o Tomás, 181,5 h.).

Las razones para el alargamiento del itinerario pueden resumirse en cuatro: período largo en orientación y pre taller; bloqueo en el aprendizaje debido a problemas emocionales; ritmo lento y carencias formativas previas.

Los procesos cortos responden principalmente a los jóvenes con niveles más elevados de decisión, autonomía y motivación, como Pedro o Mohamed. Pero también pueden responder a las situaciones de mayor dificultad en el centro: Es el caso de Tomás, a quien se facilitó la inserción laboral en una empresa en un ámbito profesional no relacionado con la formación que realizaba en el programa.

(b) Sobre los contenidos de la formación y el aprendizajes. Los contenidos de la formación responden a la visión integral del joven y a la relación que se da entre formación técnica y formación en competencias básicas o capacidades esenciales, en terminología del propio centro. Dichos contenidos, así como los aprendizajes realizados están presentes en las narraciones, y se dividen en cuatro grupos:

- Profesionales: relacionados con la profesión objeto de aprendizaje o con la búsqueda y mantenimiento del empleo.

- Educación básica: contenidos propios de la educación en materias instrumentales y alfabetización informática.

- Hábitos y comportamientos interesantes para la vida de la población joven 
(capacidades para la vida en terminología del centro): hábitos saludables (alimentación, deporte, consumo de drogas,...), sexualidad,...

- Desarrollo personal: autoconcepto, autoestima, resiliencia.

Algunos, como José, destacan los aprendizajes profesionales y consideran que lo más importante es que "sales con un oficio, aprendiendo un oficio". Otros, como Mohamed o Rosa, destacan los aprendizajes transversales relacionados con el empleo, y los realizados en su vida personal:

"Me hizo cambiar lo de ser ya jovencito, lo de comportarse bien con la persona adulta, y buscarse la vida, poder buscar la vida. [...]. No solamente te enseñan lo de trabajar, sino también como tienes que comportarte, como tienes que ser en el trabajo, como tienes que trabajar" (Mohamed).

"Considero que no ha sido una pérdida de tiempo lo de Jovent y no solo a nivel de aprender no, no. A nivel general me ha cambiado en otros aspectos de mi vida y formas de ver las cosas, y saber comportarme, tener educación" (Rosa).

(c) Sobre las estrategias metodológicas basadas en el aprendizaje autónomo y potenciadoras de dinámicas de éxito, con un componente práctico. Las narraciones reflejan un buen conocimiento y apropiación de las estrategias metodológicas del centro. El alumnado valora principalmente los aspectos relacionados con la práctica y la adecuación a los ritmos individuales de aprendizaje.

"Cada uno lleva su ritmo de trabajo. Por ejemplo, este va por, yo voy por el proyecto uno, el otro por el proyecto dos, el otro por el proyecto tres, y aparte de eso, hay trabajos a parte (sic). Por ejemplo, te hacían desmontar una caja de cambios, montarla y si había un coche para hacerle un cualquier cosa (sic), pues lo hacíamos. Te daban la oportunidad de trabajar de algo que ya has hecho. Pa saber, para yo saber, si a ti te ha quedado claro o no (sic), lo que has hecho al principio" (Mohamed). El análisis de estas narraciones indica que el alumnado valora las estrategias metodológicas con un componente práctico que potencian el aprendizaje a partir de las relaciones entre teoría y práctica, de una forma autónoma basada en el éxito en todas las fases. En este sentido, se trata de una metodología que enseña a aprender.

"Hacíamos la práctica, luego eso era lo que a mí me gustaba, vale tenías que estar ahí estudiando pero luego tú ya sabías que venía la práctica ¿de lo que habías estudiado? ${ }^{4}$ Claro. Luego tenías que hacer todo lo que tú has leído, te han explicado los pasos y todo pues tenías que hacer tú la práctica. ¿Y luego te hacian exámenes? Sí, tenía el examen después, después de haber hecho el teórico y luego el práctico pues venía el examen" (Marcial).

(d) Sobre el vínculo afectivo. Las narraciones son ricas en referencias positivas al profesorado, incluso cuando se reconoce que ha habido algún conflicto. En muchos casos, se expresa con clariddad la vinculación afectiva establecida con profesionales del centro. Marta considera que los profesores y educadores eran muy cercanos "Éramos como casi, no familia, pero casi (sic)" y estaban más "encima" del alumnado: les explicaban hasta que lo aprendieran. En este aspecto, la entrevistada remarca la diferencia entre los centros educativos en los que había estado y Jovent:

"A lo mejor en Jovent estaban más encima, ¿sabes? A lo mejor no me salía algo y me estaban encima hasta que me salía. Y en la escuela, pues si te sale o no te sale pues bueno. No pasa nada, ¿sabes? Como si pasasen [...]. La verdad es que los profesores lo hicieron "chapeau". En mi opinión lo hicieron muy bien" (Marta).

(e) Sobre el entorno educativo. El centro es valorado como un entorno favorable al desarrollo y el aprendizaje. Tomás recuerda su sorpresa cuando el primer día se encontró que "supuestamente éramos todos los peores

$4 \quad$ En itálica figura el texto que corresponde a la persona que hace la entrevista.

5 Término francés que, en el contexto de la frase, puede traducirse por "muy bien". 
de las clases juntos en la misma clase y yo me estaba quedando loco [...] todos estábamos tan tranquilos ¡no me lo puedo creer!”.

Otros, como José y Marta señalan el buen ambiente de grupo que se producía en el taller y el hecho de compartir espacios de recreo y celebraciones con los chicos y chicas de otros talleres.

Estos elementos, considerados en su totalidad, les llevan a reafirmarse cada vez más en el sentido que la realización del itinerario tiene en su proyecto personal y profesional. Este hecho, junto con el sentimiento de que son capaces de hacerlo y de sentirse a gusto en el centro, son los factores clave que determinan comportamientos que llevan a la perseverancia y al éxito.

Figura 1. Condiciones que llevan a la perseverancia y al éxito.

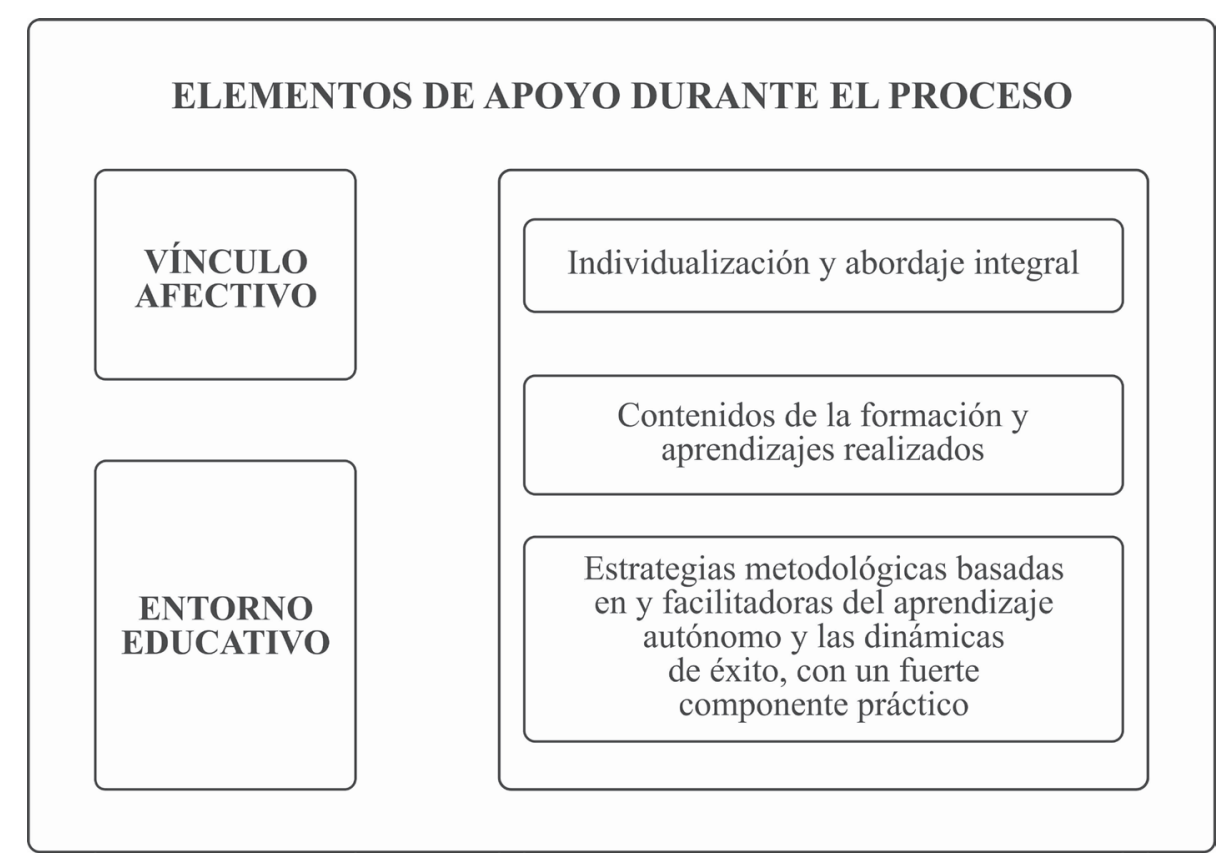

En síntesis, realizan el itinerario completo porque tiene sentido en su proyecto personal y profesional, se sienten capaces de hacerlo y se encuentran "a gusto" en el centro.

\section{Las condiciones facilitadoras del abandono}

El análisis de los casos de abandono antes de finalizar el itinerario muestra dos situaciones: (a) la de quienes abandonan a pesar de considerar que el itinerario tiene sentido en su proyecto personal y profesional, se sienten capaces de hacerlo y se encuentran a gusto en el centro (dos casos); y (b) la de quienes abandonan por no encontrar sentido al proyecto, no encontrarse a gusto o verse incapaces de finalizarlo (tres casos).
El desencadenante del abandono en el primer grupo fue, en un caso, un incumplimiento de las normas que implicaba la expulsión y, en otro, un elemento externo al joven y a la entidad: La política institucional en relación con menores inmigrantes no acompañados que prioriza el empleo a partir de los 18 años.

Las características de los jóvenes que realizan el itinerario con éxito: Una tipología en función de la vulnerabilidad personal y educativa

El análisis realizado permite establecer una tipología en función de la vulnerabilidad personal y educativa de los jóvenes que perseveran y tienen éxito en la realización del itinerario. Los dos ejes de vulnerabilidad considerados 
fueron la vulnerabilidad educativa y personal. En cada uno de ellos, se establecieron cuatro niveles, siendo 1 el de menor vulnerabilidad y 4 máxima vulnerabilidad. Una primera definición de los niveles y la ubicación de los jóvenes en las tipologías que resulta del cruce de ambos ejes, se realizó a partir de las informaciones obtenidas en las entrevistas biográficas, los documentos del centro y la información de la persona referente. Esta primera propuesta fue debatida en el grupo de discusión. De este debate surgió la siguiente propuesta de niveles de vulnerabilidad en los ejes, y de ubicación de los jóvenes en los cuatro tipos que se derivan de ello.

Los niveles de vulnerabilidad educativa se establecieron a partir de la biografía escolar y la valoración inicial realizada por el centro sobre la situación académica, las actitudes en situación de aprendizaje y la dificultad en situación de aprendizaje. De menor a mayor vulnerabilidad, son los siguientes:

- Nivel 1:Título de GESO. Se corresponde con nivel medio o alto en la valoración inicial del centro en cuanto a aspectos académicos.

- Nivel 2: Con título de GESO en diversificación o con asignaturas suspendidas o sin título de GESO pero con inicio de dificultades escolares en secundaria. Se corresponde con un nivel medio o alto en la valoración inicial del centro respecto a aspectos académicos.

- Nivel 3: Sin título de GESO. Inicio de dificultades escolares en primaria. Nivel medio o bajo de la valoración inicial del centro en cuanto a aspectos académicos.

- Nivel 4: Sin título de GESO. Escolarización breve en otro país, absentismo crónico, trayectoria errática desde primaria, último curso consolidado inferior a primaria. Nivel bajo en de la valoración inicial del centro en cuanto a aspectos académicos. En función de la vulnerabilidad personal a la entrada en el proyecto se establece un continuo que va de menor a mayor vulnerabilidad. Los elementos de vulnerabilidad personal

Rev.latinoam.cienc.soc.niñez juv 14 (2): 1405-1419, 2016 considerados son los siguientes: problemática familiar, inmigrantes extracomunitarios, situaciones de violencia, problemas de salud (física y mental), consumo de drogas, beneficiario de servicios sociales, no aceptación de su situación, otros aspectos. En el nivel inferior de vulnerabilidad no se presenta ningún elemento. En los niveles 2 y 3 se sitúan respectivamente quiénes presentan uno o dos. El nivel 4 implica tres o más.

La situación en relación con estos ejes de vulnerabilidad permitió diferenciar cuatro tipos. Las situaciones extremas están representadas por los tipos 1 y 4 . El tipo 1 está constituido por quienes presentan los niveles inferiores de vulnerabilidad educativa y personal y el 4 los niveles más elevados. En situaciones intermedias se hallan el tipo 2, con elevada vulnerabilidad educativa y baja vulnerabilidad personal y el 3 con baja vulnerabilidad educativa y elevada vulnerabilidad personal. 
Figura 2. Tipología en función de la vulnerabilidad personal y educativa.

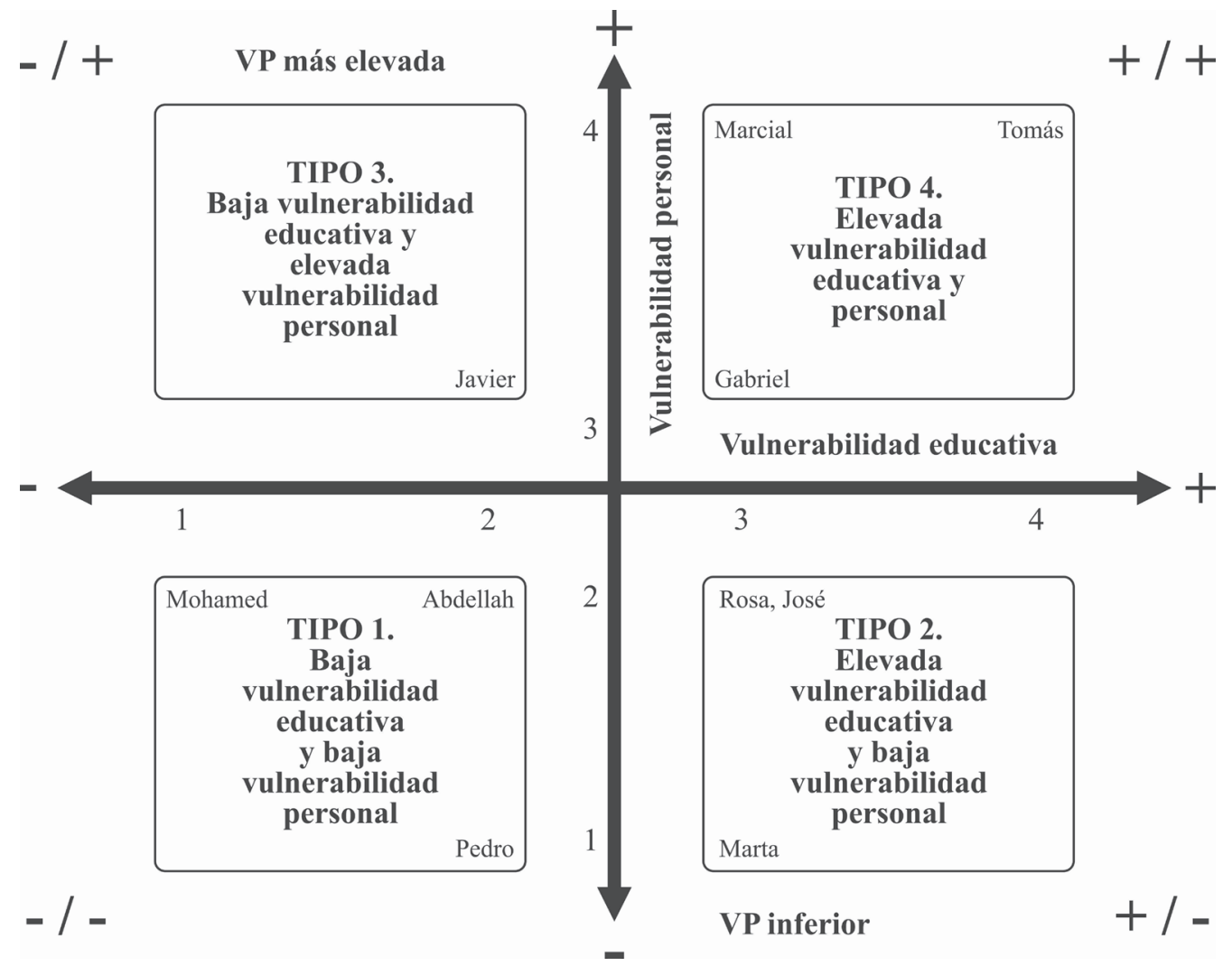

VP: Vulnerabilidad Personal.

Como se observa en la figura anterior, todos los grupos están conformados por tres personas, excepto el de baja vulnerabilidad educativa y elevada vulnerabilidad personal, en el que hay una.

\section{El impacto de la realización del itinerario}

La influencia del paso por Jovent en la situación de los y las jóvenes considerados globalmente se da de diversas formas y con distintos grados de intensidad. El caso con mayor relevancia es el de Marcial:

"Sí, cuando dejé la secundaria mi futuro era negro. [...] Yo, la verdad es que cuando salí de la escuela, yo me veía que iba a ser un vagabundo. Tirao en la calle (sic). Eso es lo que me veía en el futuro. Que iba a ser un vagabundo, ahí tirao en la calle (sic), sin trabajo, sin poder comer ni nada".

La realización del itinerario integral, además de aportarle una formación técnica y una primera experiencia laboral tuvo efectos terapéuticos. Marcial trabajó el duelo de la muerte de su padre y su falta de control, con la ayuda de la psicóloga del centro.

"Y algunos momentos que yo me estresaba mucho ahí por los problemas que había tenido, pues tenía que irme rápido para la psicóloga. ¿Te ayudaba? Me ayudaba y me hacía tranquilizarme, a lo mejor iba donde yo me estaba fumando un cigarro y se ponía a hablar conmigo, hacía como que me entendía porque me pegaba un ataque de nervios, me ponía a temblar, me ponía blanco de los nervios que pillaba y claro y yo he llegado a ser en la escuela muy agresivo y pues luego ya ahí en el Jovent me han enseñado a controlarme, a respirar hondo que eso nunca lo había hecho yo, pensar hasta diez."

Este joven continúa su relación con el centro $y$, a través del mismo, ha encontrado el trabajo que realiza en el momento de la entrevista. Después de una primera experiencia laboral como aprendiz de mecánica en la 
empresa en la que había realizado las prácticas, lo dejó porque no le gustaba el ambiente y necesitaba más dinero para mantenerse a él y a su madre. Estuvo trabajando como peón de albañil durante poco más de un año, pero la disminución de la actividad en el sector lo llevó al desempleo. Entonces acudió al centro para que le ayudaran a encontrar empleo:

"Y mi solución fue pues ir al Jovent. Fui allí y hablé con mi maestro de mecánica, hablando con él para ver si me encontraba algún trabajo y con mi tutora. Luego ya me metí en la bolsa de trabajo y me lo encontró. [...] Me encontró allí mi trabajo, me lo encontró enseguida."

Desde una perspectiva global, se destaca la mejora del autoconcepto y la autoestima que supone, en el contexto de las biografías personales y escolares de estos y estas jóvenes, el hecho de darse cuenta de que son capaces de aprender, de finalizar con éxito una formación y de hacerlo en un entorno en el que se les da confianza, se establece un vínculo afectivo, se les acepta por lo que se hace allí y no hay reproches por el pasado o por lo que se hace fuera del centro. Igualmente, se resalta la superación de problemas de comportamiento (falta de autocontrol y resolución violenta de conflictos), la disminución del consumo de estupefacientes, la mejora de las relaciones con la familia y la adaptación a la cultura del país de acogida (idioma, comportamientos) en la población inmigrante.

La influencia en relación con la inserción laboral se establece, en un primer momento, por la evaluación positiva del itinerario que implica evaluación positiva de competencias técnicas y de competencias básicas y transversales y por la realización de las prácticas profesionales. Estas van seguidas habitualmente por un contrato laboral de mayor o menor duración con la empresa en la que se han realizado las prácticas o con otra del sector. Para una parte importante de jóvenes, es la primera experiencia laboral.

El análisis longitudinal permite establecer tres tipos de trayectorias laborales en función de la relación entre la formación profesional cursada en Jovent y los trabajos realizados al finalizar el itinerario. (a) Trayectorias caracterizadas por la influencia de la formación profesional en el itinerario: es la situación más habitual, que afecta a seis de los diez jóvenes que finalizaron el itinerario. De estos, dos trabajan en la misma empresa en la que realizaron las prácticas; dos trabajan en la misma profesión o en una profesión relacionada en una empresa distinta; y otros dos no trabajan en el momento de la entrevista pero han trabajado hasta hace poco (4 meses y 1 mes respectivamente) en la misma empresa de prácticas o en la misma profesión en otra empresa.

(b) Trayectorias caracterizadas por la participación continuada en el empleo en una ocupación no relacionada con la formación profesional realizada en Jovent: Es la situación de dos jóvenes que obtuvieron evaluación positiva en competencias técnicas y transversales. En ambos casos, el hecho de tener un empleo no relacionado con la formación profesional realizada está muy ligado a su rol en la familia. Así, Marta trabaja en un hornotienda familiar en el que tiene un papel central y Mohamed dejó su empleo como aprendiz de chapista (profesión que aprendió en Jovent) para hacer de camarero, para poder aportar un mayor salario a su familia.

(c)Trayectoriasinestablesycondificultades: Dos jóvenes que han realizado itinerarios cortos y han obtenido una evaluación positiva en uno solo de los ámbitos de competencia (técnicos o transversales).

En el grupo de jóvenes que abandonaron el itinerario antes de finalizarlo, la influencia del paso por Jovent se da principalmente en los dos que no finalizaron a pesar de que tenía sentido en su proyecto personal y profesional, se sentían capaces de hacerlo y se encontraban a gusto en el centro (Isidro y Mustafá). La influencia se expresa en sus narraciones y está en la línea de lo descrito en el apartado anterior en relación con quienes finalizaron el itinerario, aunque el impacto es menor y no incluye la influencia derivada del éxito que supone la finalización con evaluación positiva. En Isidro, una parte de los empleos que tiene después de salir de Jovent están relacionados con la formación obtenida.

¿Qué te aportó tu paso por Jovent?

"Me hizo más responsable, la verdad. (...) 
Sí, porque... la verdad es que yo fumaba mucho ¿sabes? No tabaco, sino porros ${ }^{6} \mathrm{y} \ldots$ sí, podía estar todo el día en la calle, me tiraba todo el día en la calle. Y... pasaba de todo y... allí al menos me ayudaron... vi que me picaba aprender algo. [...] en casa de mi madre me lo decían, que me veían mucho mejor."

A ello hay que añadir un impacto positivo en sus hábitos, también apreciado por su madre.

\section{Conclusiones}

Los datos obtenidos han permitido desentrañar las razones por las cuales jóvenes que han salido del sistema educativo sin titulación participan en un dispositivo de ESOP en el ámbito de la formación para el empleo. También han permitido identificar las condiciones que llevan a la perseverancia y al éxito o bien al abandono. Por otra parte se ha podido elaborar una tipología de jóvenes que concluyen con éxito el itinerario formativo, y un análisis del impacto de la participación en este.

Respecto a las razones de la participación, los datos confirman resultados de investigaciones anteriores (Salvà-Mut et al., 2001, Salvà-Mut, 2007) y destacan el valor de la experiencia positiva de personas del entorno próximo de los jóvenes y el no haber podido acceder a otro dispositivo o la obligación de participar derivada de medidas judiciales o de inmigración.

En relación con las condiciones que llevan a la perseverancia y al éxito, aunque en investigaciones anteriores ya habían aparecido como elementos clave la vinculación con el profesorado, la flexibilidad y las estrategias metodológicas (Salvà-Mut et al., 2001, SalvàMut, 2007, 2009b), los datos obtenidos facilitan una visión global de las condiciones de éxito, y de las que llevan al abandono.

La heterogeneidad del alumnado y de los procesos educativos que les han conducido a la perseverancia y al éxito permiten establecer, a partir de la intersección entre un eje de vulnerabilidad personal y un eje de vulnerabilidad educativa, cuatro categorías de participantes con diferentes tipos y niveles

$6 \quad$ Término popular con el que se denominan los cigarrillos hechos de forma manual con marihuana o haschis. de vulnerabilidad y, por tanto, con diferentes necesidades educativas.

La aplicación de esta tipología facilitará la adaptación de los itinerarios a las necesidades de los participantes. Su validación con datos de otros grupos -trabajo en el que estamos inmersos- proporcionará una mayor utilidad y fiabilidad a la tipología.

El análisis del impacto que ha supuesto la participación en el itinerario refleja que este se ha producido en varias esferas de la vida de los participantes y con distintos grados de intensidad. Los resultados muestran claramente el impacto positivo que, incluso en una parte de los que abandonan el programa sin finalizarlo, ha tenido su participación en este dispositivo de ESOP.

Coherentemente con resultados anteriores (Albert et al., 2008, European Union, 2013, García et al., 2013, Marhuenda \& Navas, 2004, Marhuenda et al., 2004, Salvà-Mut et al., 2015, Salvà-Mut \& Sureda-Negre, 2012) los ahora obtenidos revelan una serie de cuestiones para el desarrollo de programas de formación para el empleo en el ámbito de la ESOP:

(a) La necesidad de itinerarios flexibles. Los diversos grados de vulnerabilidad educativa, personal y social requieren de una enorme flexibilidad en cuanto a tiempos y dispositivos. También son necesarios análisis más profundos que permitan establecer tipologías de los participantes y de las intervenciones.

(b) El rol central del trabajo psicopedagógico y delos procesos de orientación y acompañamiento. Gracias al trabajo psicopedagógico obtienen aprendizajes -como resolver de forma no violenta los conflictos o recuperar la autoestima- imprescindibles para su desarrollo personal. Y los procesos de orientación y acompañamiento son los que les apoyan en la construcción y desarrollo de un proyecto personal y profesional propio.

(c) La adaptación a los cambios en la transición de la educación al empleo. Dadas las características del mercado de trabajo y la necesidad de formación a lo largo de la vida, los apoyos que de manera informal se brindan a los jóvenes que ya han salido del programa, deberían formalizarse como servicios específicos de orientación y acompañamiento. 
(d)Lainsuficienciadelossistemashabituales de evaluación. Los resultados documentan la necesidad de superar la concepción binaria éxito-fracaso, ligados a la situación de empleo algunos meses después de finalizar la formación. También expresan la necesidad de desarrollar un sistema de indicadores de impacto centrados en el desarrollo personal, profesional y social, y su incorporación a los sistemas de evaluación. Estos indicadores deberían plantearse desde una perspectiva longitudinal e incluir como mínimo tres dimensiones: Éxito objetivo, éxito subjetivo e impacto global.

(e) El comportamiento de determinados sectores de empleo y los efectos de la crisis dificultan la inserción laboral y la autonomía de los y las jóvenes. En este contexto, se hace todavía más patente la necesidad de una red de servicios y proyectos de calidad a nivel territorial que faciliten el desarrollo personal, social y profesional de la población joven con bajo nivel educativo.

(f) La necesidad de desarrollar programas dirigidos a mejorar la participación de las mujeres: destacan en primer lugar las limitaciones al conocimiento de la problemática derivadas de que solo dos de las entrevistadas son mujeres, pero estas características de la muestra de informantes reflejan una parte de la problemática de las jóvenes con bajo nivel educativo: opciones de formación y empleo más reducidas que las de sus colegas varones y muy perjudicadas por la segregación horizontal del empleo y la formación; relación con el empleo muy influenciada por las exigencias derivadas de los roles tradicionales de género; y precarización añadida en profesiones tradicionalmente femeninas.

Los resultados, conclusiones y propuestas derivados de esta investigación han sido presentados y debatidos en varias sesiones de trabajo con el equipo de gestión del centro y en una sesión de formación con el equipo educativo. En coherencia con la perspectiva de investigación-acción, los resultados de este estudio han derivado en el desarrollo de otra investigación, que se centra en las tipologías según nivel y tipo de vulnerabilidad.

Dadas las características de la investigación, entendemos que contribuye a profundizar en el conocimiento de la ESOP y, por tanto -y en el contexto de las limitaciones en cuanto a la transferibilidad propias de la investigación cualitativa- aporta elementos de interés para profundizar en el conocimiento y la mejora de dispositivos similares.

Destaca también su potencial contribución a la construcción de políticas basadas en evidencias científicas. Las restricciones en los presupuestos destinados a políticas públicas han supuesto un incremento en los controles burocrático-administrativos de este tipo de programas, de forma que se ha rebajado el porcentaje de ausencias admisibles sin causar baja y se ha incrementado la exigencia de resultados en términos de empleo. Todo ello, sin tener en cuenta las características y las situaciones de los participantes al entrar al programa, ni la debilidad de la oferta de empleo derivada de la recesión económica. Estas exigencias suponen una fuerte presión a las entidades sociales para que seleccionen a la población menos vulnerable -en contra de los propios principios y razón de ser de la ESOPcon el fin de asegurar unos resultados exigidos para su financiación y, por tanto, para su propia supervivencia. Entendemos que investigaciones como la que presentamos expresan las debilidades de estos discursos tecnocráticos que priman eficacias supuestamente neutrales, pero que, en realidad, no sólo no promueven, sino que obstaculizan las prácticas socioeducativas que son oportunidades para los y las jóvenes sin ninguna titulación.

\section{Lista de referencias}

Abdala, E. (2004). Manual para la evaluación de impacto en programas de formación para jóvenes. Montevideo: Cinterfor.

Albert, C., Davia, M. A. \& Toharia, L. (2008). Exclusión social y pobreza: Transición educativo-formativa e inserción laboral de la población joven. Recuperado de: http:// www.seg-social.es/prdi00/groups/public/ documents/binario/115788.pdf

Albert, C. \& Davia, M. A. (2011). Pobreza monetaria, exclusión educativa y privación material de los jóvenes. Revista de Economía Aplicada, XIX (56), pp. 59-88. 
Argüello, A. (2014). La perspectiva biográfica en la investigación educativa. Una mirada a sus antecedentes, tendencias y posibilidades. Estudios Pedagógicos, XL (1), pp. 293-308.

Bertaux, D. (1997). Les récits de vie. Perspective ethnosociologique. Paris: Nathan.

Bertrand, H. (2005). Quand l'école est finie... Premiers pas dans la vie active de la Génération 2001. Marseille:Centred'Études et de Recherches sur les Qualifications (Céreq). Recuperado de: http://www.cereq. fr/enquetegeneration2004suite.htm

Bouder, A., Cadet, J. P. \& Demazière, D. (1994). Évaluer les effets des dispositifs d'insertion pour les jeunes et les chômeurs de longue durée. Un bilan méthodologique. Marseille: Centre d'Études et de Recherches sur les Qualifications (Céreq).

Comisión de las Comunidades Europeas (1995). Libro Blanco sobre la educación y la formación. Enseñar y aprender-hacia la sociedad cognitiva. Bruselas: Comisión de las Comunidades Europeas.

Demazière, D. \& Dubar, C. (1994). L'insertion professionnelle des jeunes de bas niveau scolaire. Trajectoires biographiques et contextes structurels. Marseille: Céreq.

Depover, C., Karsenti, T. \& Komis, V. (2011). La recherche evaluative. En T. Karsenti \& L. Savoie-Zajc (eds.) La recherche en éducation. Etapes et approches, (pp. 213228). Montréal: Éditions du Renoveau Pédagogique, Inc.

Desmarais, D. (2009). El enfoque biográfico. Cuestiones Pedagógicas, 20, pp. 27-54. Recuperado de: http://institucional.us.es/ revistas/cuestiones/20/art_02.pdf

European Commission (2001). Second chance schools. The results of a European Pilot Project. Luxembourg: Office for Official Publications of the European Communities.

European Union (2013). Preventing Early School Leaving in Europe-Lessons Learned from Second Chace Education. Luxembourg: Publications Office of the European Union.

Fernández-Enguita, M., Mena, L. \& Riviere, J. (2010). Fracàs $i$ abandonament escolar a Espanya. Barcelona: Fundació "La Caixa".
García, M., Casal, J., Merino, R. \& Sánchez, A. (2013). Itinerarios de abandono escolar y transiciones tras la Educación Secundaria Obligatoria. Revista de Educación, 361, pp. 65-94. Doi: 10.4438/1988-592X-00348082-RE.

García-Montalvo, J. \& Peiró, J. M. (2011). Crisis económica e inserción laboral de los jóvenes: Resultados del Observatorio de Inserción Laboral de los Jóvenes 2011. Valencia: Instituto Valenciano de Investigaciones Económicas. Doi: $\quad$ http://dx.doi.org/10.12842/ OBSERVATORIO_2011.

Häfeli, K. \& Schellenberg, C. (2009). Facteurs de réussite dans la formation professionnelle des jeunes à risque. Berne: Conférence suisse des directeurs cantonaux de l'instruction publique (Cdip).

Marhuenda, F. \& Navas, A. (2004). Replantear la garantía social. Ventajas y problemas tras diez años de experiencias. Valencia: Universitat de València.

Marhuenda, F., Navas, A. \& Pinazo, S. (2004). Conflicto, disciplina y clima de aula: La garantía social como respuesta al control social sobre los jóvenes. En M. Molpeceres (ed.) Identidades y formación para el trabajo, (pp. 255-299). Ginebra: Oficina Internacional del Trabajo, Cinterfor.

Nicole-Drancourt, C. (1991). Le Labyrinthe de l'insertion. Paris: La Documentation Française.

OECD (2013). Education at Glance 2013: OECD Indicators. Paris: OECD.

Salvà-Mut, F. (2007, septiembre). La coévaluation en tant qu'élement d'amélioration des projets de formation et d'insertion professionnelle pour les jeunes de bas niveau scolaire. Ponencia presentada en Université d'Été Européenne de la Recherche et des Innovations pour Apprendre tout au Long de la Vie. Luxemburgo.

Salvà-Mut, F. (2009a, mayo). La necesidad del programa para favorecer $y$ dar oportunidades al alumnado en riesgo de exclusión social. Ponencia presentada en Jornada Programas de Cualificación 
Profesional Inicial, Palma de Mallorca, España.

Salvà-Mut, F. (2009b). Estrategias de innovación en la formación de personas con bajo nivel educativo: Otras miradas. En J. Tejada (ed.) Estrategias de innovación en la formación para el trabajo, (pp. 143156). Madrid: Tornapunta Ediciones, SLU.

Salvà-Mut, F., Calvo-Sastre, A. \& Cloquell, A. (2001). La investigación en el ámbito de la evaluación de proyectos de inserción sociolaboral para jóvenes con bajo nivel de cualificación. Revista de Investigación educativa, 19 (2), pp. 529-541.

Salvà-Mut, F., Oliver-Trobat, M. \& CalvoSastre, A. (2006). Young people of low educational level: Promoting innovative projects. European Journal of vocational training, 38 (2), pp. 49-65.

Salvà-Mut, F., Quintana-Murci, E. \& Desmarais, D. (2015). Inclusion and exclusion factors in adult education of youth with a low level educational level in Spain. European journal for Research on the Education and Learning of Adults, 6 (1), pp. 9-23. Doi 10.3384/rela.2000-7426.rela0121.

Salvà-Mut, F. \& Sureda-Negre, J. (2012). L'utilisation des histoires de vie dans la recherche et l'innovation de l'école de la deuxième chance. En D. Desmarais, I. Fortier \& J. Rhéaume (ed.) Transformations de la modernité et pratiques (auto) biographiques, (pp. 147-160). Québec: Presses de 1'Université du Québec.

Sen, A. (2000). Social Exclusion: Concept, Application, and Scrutiny. Manila: Asian Development Bank.

Silver, H. \& Miller, S. M. (2003). Social Exclusion:TheEuropeanApproach to Social Disadvantage. Indicators, 2 (2), pp. 5-21. Doi: 10.1080/15357449.2003.11069166. 